Proc. XIX International School of Semiconducting Compounds, Jaszowiec 1990

\title{
PHOTO-EPR INVESTIGATIONS OF THERMAL DONORS IN SILICON*
}

\begin{abstract}
N.T. BAGRAEV,
A.F. Ioffe Physico-Technical Institute, Leningrad, 194021, U.S.S.R.

H.H.P.Th. Bekman, T. Gregorkiewicz and C.A.J. AmmerlaAn

Natuurkundig Laboratorium der Universiteit van Amsterdam, Valckenierstraat 65, NL-1018XE Amsterdam, The Netherlands

(Received August 8, 1990)

Two thermal donor related EPR centers Si-NL8 and Si-NL10 were studied by the photo-EPR technique. The spectral dependence of the intensity of the EPR signals was determined for the region from 0.6 to $1.4 \mathrm{eV}$ in several samples with different Fermi level position. The quenching of the Si-NL8 signal with the coinciding enhancement of the Si-NL10 signal has been observed with possible indication of some metastable behavior of the NL8 center. For the heavily aluminum doped material strong generation of photocurrent upon band gap illumination has been observed. The results of the study provide further evidence for mutal correlation of both thermal donor related EPR centers since the observed spectral dependences could be understood by relating both NL8 and NL10 spectra to different paramagnetic states of basically the same center.
\end{abstract}

PACS numbers: $76.30 .-\mathrm{v}, 72.20 . \mathrm{Jv}$

\section{Introduction}

Thermal donors are oxygen-related centers of shallow, effective mass, donor character which are created upon annealing in oxygen-rich crystals of silicon and germanium. In spite of the fact that the phenomenon has been already known for a long time, the physics of thermal donor centers remains full of unanswered

*This work has been supported by the Netherlands Foundation for Fundamental Research on Matter FOM. 
questions [1]. Among them especially puzzling are those related to the atomic configuration and the transformation mechanism of thermal donors.

Quite recently the thermal donor issue was further complicated by the discovery of the bistability effect [2-4]. According to those findings some of the thermal donor centers (early species TD1 and TD2 for silicon, and D, E and F for germanium, can exist in two different structural configurations depending on Fermi level position. In one of the configurations the center has a well known shallow double donor character while the other one is connected to a deep mid-gap energy level. The phenomenon was discovered in infrared absorption studies as some of the transitions could only be observed when the sample was cooled under band gap illumination. Following that observation the bistability effects were also investigated by electron paramagnetic resonance (EPR).

Following the identification of EPR spectra related to thermal donors in silicon [5, 6] and germanium [7] a vast amount of detailed information on structure and symmetry of these centers was obtained as the EPR studies could further be augmented by the more advanced ENDOR techniques [8-10]. This was especially welcomed in view of the importance of the thermal donor issue in submicron technology of silicon. The application of the magnetic resonance studies was, however, until now restricted only to the shallow configuration of thermal donors. It is the aim of this paper to pursue the possible application of the EPR technique also to the deeper stable configuration.

\section{Results and discussion}

Following the aim of the paper the photo-EPR technique has been applied. The EPR measurements were performed in the $K$ microwave band (23 GHz) and the dispersion part of the signal was followed. The sample placed in the microwave cavity at liquid helium temperature could be illuminated by monochromatic light. A phosphorus-doped silicon crystal was simultaneously present in the cavity in order to provide the calibration standard for the comparison of the signal intensity. The material used in the study was Czochralski-grown silicon with various doping levels so as to provide different Fermi level positions. The samples were heat-treated at $470^{\circ} \mathrm{C}$ in order to generate thermal donor centers whose presence was manifested by NL8 and NL10 EPR spectra.

In the first approach the EPR spectrum was measured for the specimen cooled in darkness and under band gap illumination. In case of germanium in an experiment like this, a clear generation of a new EPR spectrum for the illuminated sample could be concluded [11]. However, in the present experiment for silicon samples only the change of the EPR signal intensity was observed yielding therefore a less reliable result. In order to obtain more information, the dependence of the EPR signals on the illumination was studied as a function of the wavelength. The results of such investigation with the calibrated intensity of the EPR signals are shown in Fig. 1. In Fig. 1(a) the dependence as obtained for the originally $p$-type, aluminum doped Cz-Si sample is shown. In such material both EPR spectra NL8 and NL10 can be observed following the annealing procedure of 7 hours. As can be 
(a)

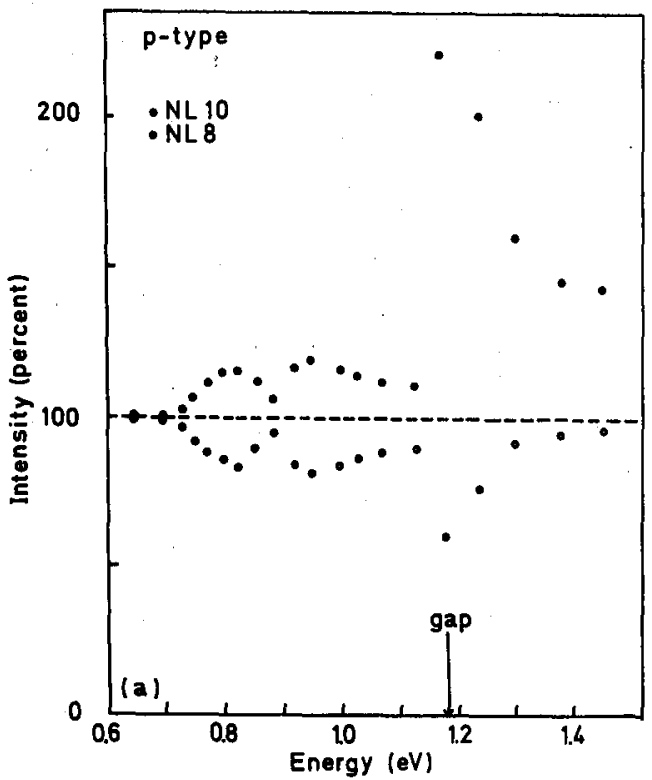

(b)

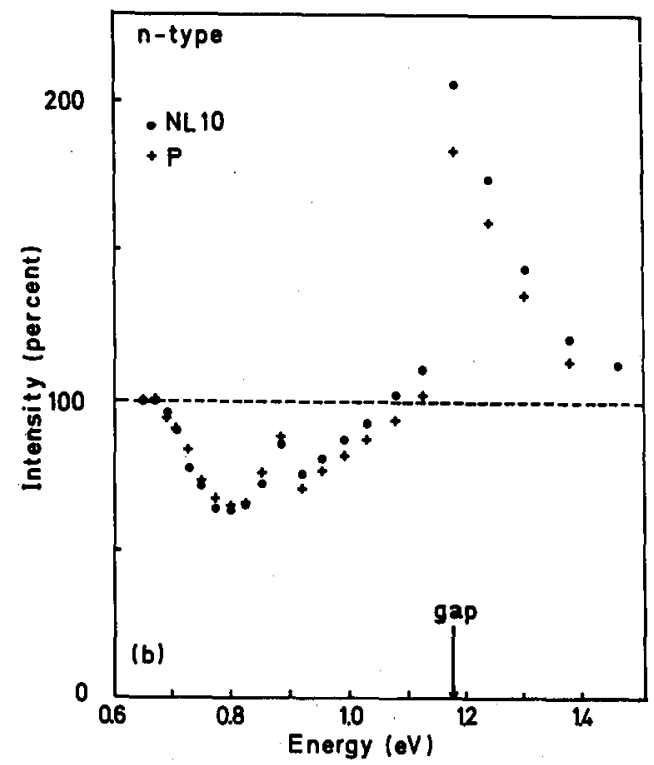

Fig. 1. Spectral dependence of the photo-EPR signal of thermal donor related NL8 and NL10 centers in (a) Cz-Si, originally p-type, heat treated for $7 \mathrm{hrs} / 470^{\circ} \mathrm{C}$ and (b) $\mathrm{Cz}-\mathrm{Si}$, originally $n$-type, heat treated for $15 \mathrm{hrs} / 470^{\circ} \mathrm{C}$. 
seen the behavior of the two spectra appears to be anticorrelated with two clear maxima for the NL10 around 0.8 and $0.95 \mathrm{eV}$. In Fig. 1(b) a similar result but for a sample with higher positioned Fermi level is presented. The sample was originally $n$-type, phosphorus doped and was given 15 hours heat-treatment. The intensity of the phosphorus signal is also depicted, while the high position of the Fermi level prohibited the observation of the NL8 signal. As can be seen the NL10 and phosphorous signal behave in this case very similarly showing again two extrema for the same energies as before. Additionally to the experiments whose results are shown in Fig. 1, a similar study was attempted for the $\mathrm{Cz}$-Si material heavily doped with aluminum $\left(\sim 10^{17} \mathrm{~cm}^{-3}\right)$. However, in that case illumination of the sample resulted in strong conductivity increase which in turn led to substantial reduction of the quality factor of the cavity.

The results as depicted in Figs. 1(a) and 1(b) are very difficult for undisputable interpretation. Nevertheless, on the basis of the study, it can be concluded that the occupancy of two thermal donor related paramagnetic centers NL8 and NL10 is governed by two levels in the gap as evidenced by the occurence of two extrema in Fig. 1. Moreover, the same levels influence both centers. These levels are (most probably) introduced in the sample by heat-treatment (no such dependence has ever been reported for the phosphorous signal in untreated silicon samples) and do not coincide with the "normal" thermal donor levels at 60 and $120 \mathrm{meV}$ below the conduction band. Also, the (significantly different) kinetics of generation and quenching of the NL8 signal could be indicative of the metastable character of this center.

\section{References}

[1] T. Gregorkiewicz, H.H.P.Th. Bekman, Mater. Sci. Eng. B 4, 291 (1989).

[2] L.F. Makarenko, V.P. Markevich, L.I. Murin, Sov. Phys. Semicond. 19, $1192(1985)$.

[3] P. Wagner, J. Hage, Appl. Phys. A, Solid Surf. 49, 123 (1989).

[4] P. Clauws, J. Vennik, Mater. Sci. Forum 38-41, 473 (1989).

[5] S.H. Muller, M. Sprenger, E.G. Sieverts, C.A.J. Ammerlaan, Solid State Commun. 25, 987 (1978).

[6] T. Gregorkiewicz, D.A. van Wezep, H.II.P.Th. Bekman, C.A.J. Ammerlaan, Phys. Rev. B 35, 3810 (1987).

[7] F. Callens, P. Clauws, P. Matthys, E. Boesman, J. Vennik, Phys. Rev. B, Condens. Matter 39, 11175 (1989).

[8] T. Gregorkiewicz, H.H.P.Th. Bekman, C.A.J. Ammerlaan, Phys. Rev. B, Condens. Matter 38, 3998 (1988).

[9] H.H.P.Th. Bekman, T. Gregorkiewicz, C.A.J. Ammerlaan, Phys. Rev. B, Condens. Matter 39, 1648 (1989).

[10] J. Michel, J. R. Niklas, J.-M. Spaeth, Phys. Rev. B, Condens. Maller 40, 1732 (1989).

[11] H.H.P.Th. Bekman, T.Gregorkiewicz, I.F.A. Hidayat, C.A.J. Ammerlaan, P. Clauws, submitted for publication in Phys. Rev. B. 